\title{
Conglomerate Entrenchment under Optimal Financial Contracting*
}

\author{
A ntoine Faure-Grimaud ${ }^{\dagger} \quad$ Roman Inderst ${ }^{\ddagger}$
}

J uly 2004

\begin{abstract}
A bstract
We provide a formal analysis of the notion that conglomerates are more 'entrenched' as they have 'deeper pockets'. Using the financial contracting model of B olton and Scharfstein (1990), we can isolate two effects that confirm this conjecture: the pooling of cash flows, which allows to smooth out repayments, and the ability to obtain better credit terms. For less profitable business segments, the internal capital market operated in a conglomerate may, however, work in the opposite direction, increasing the sensitivity of operations to own cash flows and increasing the likelihood of exit.
\end{abstract}

Keywords: Conglomerate entrenchment; financial constraints; predation.

${ }^{*}$ B oth authors gratefully acknowledge financial support from the Financial M arkets Group (F M G). The paper benefitted from the comments of two referees and the editor, B en Bernanke.

${ }^{\dagger}$ London School of Economics \& CEPR. Address: Department of Economics, London School of Economics, Houghton Street, London WC2A 2AE. Email: a.faure-grimaud@lse.ac.uk.

${ }^{\ddagger} L$ ondon School of E conomics \& CEPR. Address: Department of E conomics \& Department of A ccounting and Finance, London School of Economics, Houghton Street, London W C 2A 2AE. E mail: r.inderst@lse.ac.uk. 


\section{Introduction}

A ccording to a widespread view, the 'deep pockets' of a conglomerate make its divisions more 'entrenched' compared to focused firms. On the back of the conglomerate's financial strength, its divisions are thought to be less likely to cut back on investments or even being shut down after low cash flows, while they are supposedly more likely to engage in (predatory) behavior aimed at harming competitor. ${ }^{1}$ This paper uses an optimal contracting model to put the notion of 'conglomerate entrenchment' to formal scrutiny. In our model, firms can access the external capital market, but agency problems with outside investors impose frictions. As it is difficult for firms to commit to repay investors out of future profits, investors keep firms on a tight leash and make the commitment of fresh funds dependent on the repayment of earlier loans.

The first part of our analysis focuses on the conglomerate's ability to pool cash flows across divisions. Cash-flow pooling can enable the conglomerate to fully repay creditors even when one division performs poorly. This provides each division with additional protection against a shortfall in own cash flows. ${ }^{2}$ The flipside for the better-performing division is that by 'crosssubsidizing' the underperforming division it risks not being refinanced itself. As a third implication of cash-flow pooling, we find that by promising investors a safer repayment a conglomerate obtains better credit terms, leading to an overall higher probability of refinancing.

We show that these three implications of cash-flow pooling all work in the same direction, reducing the sensitivity of a division's operations to own cash flows compared to a focused firm. Likewise, a focused firm is more likely to exit following low cash flows. The differences between a conglomerate's division and a focused firm are less pronounced if the agency problem with investors is less severe and financing constraints are consequently reduced.

By focusing on divisions with homogeneous reinvestment opportunities, the first part of our analysis abstracts from a second key aspect of joint incorporation. In an internal capital

\footnotetext{
${ }^{1}$ A ntitrust authorities often harbor suspicions against conglomerate mergers, fearing further 'entrenchment' in the concerned markets. A case in place is the European Commission's resistance to the GE-Honeywell merger (Case No. COMP/M.2220, J uly 2001). While the potential for 'entrenchment' has not been dismissed per se in the U.S., the view is more benign- with the possible exception of firms that may cross-subsidize predatory strategies from profits earned in regulated markets.

${ }^{2} T$ he role of 'co-insurance' to smooth out repayments and, thereby, ease financing constraints has already been documented elsewhere, e.g., in Diamond (1984), Fluck and Lynch (1999), and Inderst and M üller (2003).
} 
market, limited fresh funds are first allocated to the most profitable divisions. For less profitable divisions, 'winner-picking' can more than undo the positive implications of cash-flow pooling, increasing their cash-flow sensitivity and making exit more likely than for a stand-alone firm.

Recent evidence for 'winner-picking' comes from Maksimovic and P hillips (2002), who showed that conglomerates allocate more resources to units with better relative investment opportunities. In a similar spirit, K hanna and Tice (2001) document that diversified retail firms were quicker to react following Wal-Mart's entry into a local market, e.g., by shutting down their discount business. ${ }^{3}$

The extant theoretical literature on predation and entrenchment focuses on the more vulnerable financial structure of new entrants (e.g., Telser (1966), Benoit (1984)). ${ }^{4}$ The financial literature on competition and firm exit focuses likewise on the effects of financial leverage. (See, for instance, Kovenock and Phillips (1997) for an overview.)

The rest of this paper is organized as follows. Section 2 discusses the benchmark of a focused firm. Section 3 analyzes the implications of cash-flow pooling with homogeneous divisions. Section 4 introduces 'winner-picking' and Section 5 concludes with an application to predation.

\section{The Benchmark of a Focused Firm}

Our model is a variant of B olton and Scharfstein (BS, 1990). We consider a penniless firm that operates in only one market or industry. The firm operates for at most two periods, $t=1,2$, and requires each time a fixed investment of $F>0$. There is a competitive market for financing. Once financed, the firm realizes with probability $0<1-\theta_{t}<0$ the cash flow $\pi_{h}>0 .{ }^{5}$ The expected cash flow in $t$ is denoted by $\bar{\pi}_{t}:=\left(1-\theta_{t}\right) \pi_{h}$. We stipulate that continuing in $t=2$ is efficient: $\bar{\pi}_{2}>F$. (If $\bar{\pi}_{1}<F$, we may view $t=1$ as the 'growth period'.) The respective states of high and low (zero) cash flows are denoted by $s \in S=\{l, h\}$.

Importantly, only the fraction $\alpha$ of high cash flows is verifiable. This limits the repayment

\footnotetext{
${ }^{3}$ For benefits of 'winner-picking' see also Stein (1997). Scharfstein and Stein (2000) and Rajan, Servaes, and Zingales (2000) argue that these benefits can be muted by internal rent seeking and power struggles.

${ }^{4}$ The industrial organization literature also analyzes exit behavior of multi-plant firms in oligopolies (e.g., Ghemawat and Nalebuff (1990)). Interestingly, our result that diversification can both increase and decrease the probability of exit may help to explain the ambiguous findings in the literature (e.g., Deily (1991)).

${ }^{5} \mathrm{BS}$ set $\theta_{1}=\theta_{2}$ and consider a monopolistic investor in their main analysis.
} 
that can be promised to the investor in $t=2$ to $\alpha \pi_{h}$. However, in $t=1$ the investor can use the threat of withholding refinancing to extract more than $\alpha \pi_{h}$, but this is only incentive compatible if the firm is rewarded with a strictly higher probability of refinancing: $\beta(h)>\beta(l)$. The full contractual problem is now as follows. In $t=1$, the firm makes a repayment $R(s) \leq \pi_{s}{ }^{6}$ Conditional on the revealed state and the corresponding repayment, the lender refinances the firm with probability $\beta(s) .{ }^{7}$ The optimal contract maximizes the firm's ex-ante profits subject to the lender's break-even constraint and the firm's incentive compatibility constraints. ${ }^{8}$

Proposition 1. Under the optimal financial contract, a focused firm with high cash flows repays $R(h)<\pi_{h}$ and continues operations with probability $\beta(h)=1$. If only the fraction

$$
\alpha<2 F /\left(\bar{\pi}_{1}+\bar{\pi}_{2}\right)
$$

of cash flows is verifiable, the firm only continues with probability $\beta(l)<1$ following low cash flows. If (1) does not hold, we have $\beta(l)=1$.

Proof. The proof uses arguments from BS and is thus kept short. In $t=2$, the firm realizes $(1-\alpha) \bar{\pi}_{2}$ in case of refinancing. For $R(h)>\alpha \pi_{h}$, incentive compatibility thus requires

$$
R(h)-\alpha \pi_{h} \leq[\beta(h)-\beta(l)](1-\alpha) \bar{\pi}_{2} .
$$

The optimal contract maximizes the firm's expected profits, $\theta_{1} \beta(l)(1-\alpha) \bar{\pi}_{2}+\left(1-\theta_{1}\right)\left[\pi_{h}-\right.$ $\left.R(h)+\beta(h)(1-\alpha) \bar{\pi}_{2}\right]$, subject to (2) and the lender's break-even constraint

$$
\theta_{1} \beta(l)\left(\alpha \bar{\pi}_{2}-F\right)+\left(1-\theta_{1}\right)\left[R(h)+\beta(h)\left(\alpha \bar{\pi}_{2}-F\right)\right] \geq F,
$$

which binds by optimality. If (1) does not hold, (3) is satisfied for $\beta(h)=\beta(l)=1$ and a unique value $R(h) \leq \alpha \pi_{h}$. From $\bar{\pi}_{2}>F$ this is uniquely optimal. If (1) holds, (3) requires $R(h)>\alpha \pi_{h}$

\footnotetext{
${ }^{6}$ We thus already set the repayment in $t=2$ equal to $\alpha \pi_{h}$ or zero, depending only on the final cash-flow realization. This is without implications for our results.

${ }^{7}$ One specification is that in $t=1$ cash flows are first only privately observed by the firm. (Hence, $\beta(s)$ is conditional on the firm's message $s \in S$.) Even if the cash-flow state becomes public knowledge- i.e., after reinvesting fresh funds- the firm cannot be prosecuted for 'consuming' the fraction $1-\alpha$ of high cash flows.

${ }^{8} \mathrm{~N}$ ote that, as this is all verifiable, the optimal contract (i) forces the firm to invest the received funds, (ii) prohibits the firm from obtaining financing from other sources, and (iii) ensures that the firm indeed shuts down in case this is prescribed by the contract. In Inderst and Müller (2003) a weak legal environment prevents investors from stopping firms to operate with their hidden (or 'privately consumed') funds after defaulting on their repayment obligations. M oreover, for a discussion of renegotiations in case of forced shut-down see BS.
} 
and thus $\beta(h)-\beta(l)>0$ from (2). By $\bar{\pi}_{2}>F$ it is optimal to minimize $\beta(h)-\beta(l)$, implying that (2) binds. Substituting the binding constraints (3) and (2), we obtain $\beta(h)=1$ and

$$
\beta(l)=\frac{\left(1-\theta_{1}\right)\left(\bar{\pi}_{2}-F\right)-\left(F-\alpha \bar{\pi}_{1}\right)}{\left(1-\theta_{1}\right)\left(\bar{\pi}_{2}-F\right)+\left(F-\alpha \bar{\pi}_{2}\right)},
$$

while $R(h)<\pi_{h}$ is determined from substituting $\beta(l)$ and $\beta(h)$ into (2). Q.E.D.

If $\alpha$ satisfies (1), the lender can only break even if more than the verifiable component $\alpha \pi_{h}$ is repaid in $t=1$, which by incentive compatibility requires $\beta(h)-\beta(l)>0$. Optimally, the refinancing probability $\beta(l)$ is chosen such that the investor just breaks even. (We always assume that financing is feasible, which- as is easily shown- is the case if $\left(1-\theta_{1}\right)\left(\bar{\pi}_{2}-F\right) \geq\left(F-\alpha \bar{\pi}_{1}\right)$.) Note that $\beta(s) \leq 1$ could also represent the expansion of a scalable investment project. With some caution, we may then suppose that the firm can raise the amount $\beta(l) F$ of 'long-term finance'. Following default in $t=1$, the amount of 'short-term finance' $[1-\beta(l)] F$ is no longer rolled over. ${ }^{9}$ F inally, differentiating $\beta(l)$ in (4) yields the following result.

Corollary 1. For low values of $\alpha$ where (1) holds, $\beta(h)-\beta(l)$ decreases with $\alpha$.

Hence, if the agency problem (as measured by the non-verifiable fraction $1-\alpha$ ) becomes less severe, the firm is refinanced with higher probability and the cash-flow sensitivity, $\beta(h)-\beta(l)$, is reduced. If $\alpha$ is sufficiently high, refinancing becomes independent of first-period cash flows: $\beta(h)=\beta(l)=1$. Firms may have lower agency problems as they, for instance, operate in an environment with stronger investor protection or as their investments are more 'tangible'. ${ }^{10}$ We have more to say on the impact of $\alpha$ below when we compare focused firms with conglomerates.

\section{Conglomerates with Homogeneous Divisions}

A conglomerate operates two divisions, $i \in I=\{A, B\}$, both requiring in $t=1,2$ the investment of $F>0$. High cash flows are independently realized with probabilities $\theta_{t}^{i}$. In this section, we assume that divisions are homogeneous: $\theta_{t}^{A}=\theta_{t}^{B}=\theta_{t}$. The cash-flow states in $t=1$ are $S=\{(h, h),(h, l),(l, l)\}$ with probabilities $q(s)$ and cash flows $r(s) .{ }^{11}$ That is, $q(l, l)=\left(\theta_{1}\right)^{2}$

\footnotetext{
${ }^{9} \mathrm{~T}$ he firm is prevented from using any 'hidden' (or 'consumed') funds $(1-\alpha) \pi_{h}$ to scale up the investment.

${ }^{10} \mathrm{~A}$ monotonic relationship between measures of financial constraints and corporate investment policies has been documented by numerous papers building on the seminal work of Fazzari, Hubbard, and Petersen (1988).

${ }^{11}$ A ggregating cash flows across all divisions would be less adequate for business groups, where units often borrow separately. For an analysis of the debt capacity of business groups see Bianco and Nicodano (2002).
} 
and $r(l, l)=0, q(h, l)=2 \theta_{1}\left(1-\theta_{1}\right)$ and $r(h, l)=\pi_{h}, q(h, h)=\left(1-\theta_{1}\right)^{2}$ and $r(h, h)=2 \pi_{h}$.

The likelihood of refinancing depends again on the interim payout to investors, $R(s) \leq r(s)$. The contract specifies the probabilities with which the investor injects zero, $F$, or $2 F$ in $t=2$. Depending on the (revealed) state, we denote the probability of receiving $F$ by $\gamma_{1}(s)$ and that of receiving $2 F$ by $\gamma_{2}(s)$. No refinancing is received with probability $1-\gamma_{1}(s)-\gamma_{2}(s)$. If the firm receives less than $2 F$, it decides which project to continue. As divisions are homogeneous, they are treated identically. Consequently, each division has the refinancing probability $\beta^{i}(s)=$ $\beta(s)=\gamma_{1}(s) / 2+\gamma_{2}(s) .{ }^{12}$ In what follows, it will often be convenient to suppose that the contract directly specifies the probabilities $\beta^{i}(s){ }^{13}$

We next isolate three implications of forming a conglomerate. First, pooling cash flows across divisions allows to smooth out repayments to investors, shielding both divisions from being shut down after low own cash flows. Formally, recall from Proposition 1 that a focused firm with high cash flows repays strictly less than $\pi_{h}$, i.e., $\pi_{h}-R(h)>0$, while after low cash flows it is sometimes shut down. In the conglomerate, these 'excess' cash flows $R(h)-\pi_{h}$ can be used to make up for the underperformance of another division. ${ }^{14}$ The flipside for the high cash-flow division is that 'cross-subsidization' may reduce its own refinancing probability, which is the case if $\beta(h, l)<1$. Finally, by promising a safer repayment a conglomerate can obtain 'better' credit terms, which in our model leads to an overall higher refinancing probability, i.e., including the state where both divisions have zero cash flows: $\beta(l, l)>\beta(l)$.

Proposition 2. Under the optimal financial contract, divisions in a homogeneous conglomerate get refinanced as follows. If $\alpha$ is high such that (1) is not satisfied, we have $\beta(s)=1$ for all $s$. Otherwise, we have $\beta(h, h)=1$ and $\beta(l)<\beta(l, l)<1$, while for $\beta(h, l)$ we have two possibilities. In case $\pi_{h} \geq 2 \bar{\pi}_{2}$, it always holds that $\beta(h, l)=1$. For $\pi_{h}<2 \bar{\pi}_{2}, \beta(h, l)=1$ holds if $\alpha$ is not too low or $F$ not too high, while otherwise we have that $\beta(l, l)<\beta(h, l)<1$.

Proof. Suppose that (1) holds. (The case where it does not hold is again immediate.) As noted previously, it is convenient to suppose that contracts directly specify $\beta(s)$, which are chosen to

\footnotetext{
${ }^{12}$ In case of scalable projects, the investor would provide the aggregate funds $F\left[\gamma_{1}(s)+2 \gamma_{2}(s)\right]=2 F \beta(s)$. In this case, setting $\beta^{A}(s)=\beta^{B}(s)$ would also be uniquely optimal if we had symmetric but strictly concave investment technologies.

${ }^{13} \mathrm{~W}$ ith heterogeneous divisions the firm and the investor will both prefer to continue the most profitable project, i.e., there is no conflict of interest regarding the choice of projects.

${ }^{14} \mathrm{~T}$ his effect is also dealt with in Inderst and Müller (2003).
} 
maximize $\sum_{s \in S} q(s)\left[r(s)-R(s)+2 \beta(s)(1-\alpha) \bar{\pi}_{2}\right]$ subject to the investor's break-even constraint

$$
\sum_{s \in S} q(s)\left[R(s)+2 \beta(s)\left(\alpha \bar{\pi}_{2}-F\right)\right] \geq 2 F,
$$

the cash-flow constraints $r(s) \geq R(s)$ for $s \in S$, and the set of incentive compatibility constraints. In the Appendix, we show the following intuitive results: (i) $\beta(h, h)=1$ and $r(h, h) \geq R(h, h)$ never binds; (ii) we can restrict consideration to the incentive constraints

$$
R(s)-\alpha r(s) \leq 2[\beta(s)-\beta(l, l)](1-\alpha) \bar{\pi}_{2} \text { for } s \in\{(h, h),(h, l)\},
$$

which both bind at an optimum; and (iii) if $r(h, l) \geq R(h, l)$ does not bind we have $\beta(h, l)=$ 1. If $r(h, l) \geq R(h, l)$ does not bind (Case 1 ), we can next substitute the binding incentive compatibility constraints (6) into the binding break-even constraint (5) to obtain

$$
\beta(l, l)=\frac{\left[1-\left(\theta_{1}\right)^{2}\right]\left(\bar{\pi}_{2}-F\right)-\left(F-\alpha \bar{\pi}_{1}\right)}{\left[1-\left(\theta_{1}\right)^{2}\right]\left(\bar{\pi}_{2}-F\right)+\left(F-\alpha \bar{\pi}_{2}\right)},
$$

which by $1-\left(\theta_{1}\right)^{2}>1-\theta_{1}$ strictly exceeds $\beta(l)$ in (4). If $r(h, l) \geq R(h, l)$ binds (Case 2 ), we have from $R(h, l)=\pi_{h}$ and (6) that $\beta(h, l)=\pi_{h} /\left(2 \bar{\pi}_{2}\right)+\beta(l, l)$, which together with (6) for $(h, h)$ can be substituted into (5) to obtain

$$
\beta(l, l)=\frac{\left[\left(1-\theta_{1}\right)^{2}+\theta_{1}\left(1-\theta_{1}\right) \frac{\pi_{h}}{\bar{\pi}_{2}}\right]\left(\bar{\pi}_{2}-F\right)-\left(F-\alpha \bar{\pi}_{1}\right)}{\left(1-\theta_{1}\right)^{2}\left(\bar{\pi}_{2}-F\right)+\left(F-\alpha \bar{\pi}_{2}\right)} .
$$

Comparison with (4) reveals again that $\beta(l, l)>\beta(l) .{ }^{15}$ Next, Case 1 applies if $\pi_{h} \geq$ $2[1-\beta(l, l)] \bar{\pi}_{2}$, where $\beta(l, l)$ satisfies (7). As $d \beta(l, l) / d \alpha>0$ with $\beta(l, l) \rightarrow 1$ as $\alpha$ converges to the threshold in (1), Case 1 applies for all $\alpha$ if it does so for $\alpha=0$, while otherwise it applies if $\alpha \geq \bar{\alpha}$, where $0<\bar{\alpha}<2 F /\left(\bar{\pi}_{1}+\bar{\pi}_{2}\right)$. Note also that $d \beta(l, l) / d F<0$ in (7) and that for $\alpha=0$ we have $\beta(l, l) \rightarrow 1$ as $F \rightarrow \alpha\left(\bar{\pi}_{1}+\bar{\pi}_{1}\right) / 2$. Finally, at the (highest feasible) value $F$ where $\beta(l, l)=0$, we have for $\alpha=0$ that Case 1 applies if $\pi_{h} \geq 2 \bar{\pi}_{2}$. Q.E.D.

We briefly comment on when the two cases $\beta(h, l)=1$ and $\beta(h, l)<1$ apply. Intuitively, $\beta(h, l)=1$ holds if the financing problem is less severe as less funds are required or as a higher fraction of cash flows is verifiable. Alternatively, $\beta(h, l)=1$ always holds if $\pi_{h} \geq 2 \bar{\pi}_{2}$. Here, the intuition is that a high value of $\pi_{h}$ implies high 'excess' cash flows $R(h)-\pi_{h}$, allowing the

\footnotetext{
${ }^{15}$ If we substitute $\beta(l, l)=\beta(l)$ from (4) into (5), the investor's profits equal $2\left(\bar{\pi}_{2}-F\right)\left(1-\theta_{1}\right) \theta_{1}\left[\frac{\pi_{h}}{\bar{\pi}_{2}}-(1-\beta(l))\right]>$ 0 , implying $\beta(l, l)>\beta(l)$ to make (5) binding.
} 
conglomerate to obtain refinancing for both divisions even if only one generated high cash flows. Note next that for each division the (expected) sensitivity of operations to own cash flows equals

$$
\theta_{1}[\beta(h, l)-\beta(l, l)]+\left(1-\theta_{1}\right)[\beta(h, h)-\beta(h, l)] .
$$

The following result mirrors Corollary 1.

Corollary 2. For low values of $\alpha$ where (1) holds, an increase in $\alpha$ strictly decreases the sensitivity to own cash flows (9) for each division in a homogeneous conglomerate while it strictly increases its refinancing probability in each state (where not already $\beta(s)=1$ ).

P roof. The assertions follow as $d \beta(l, l) / d \alpha>0$ holds from (7) in case $\beta(h, l)=1$, while for $\beta(h, l)<1$ we have $d \beta(l, l) / d \alpha>0$ from (8) and $d \beta(h, l) / d \alpha=d \beta(l, l) / d \alpha$. Q.E.D.

In the remainder of this section, we compare a conglomerate to a focused firm.

Corollary 3. If (1) holds, joint incorporation in a homogeneous conglomerate strictly increases both the probability with which a business is continued following low own cash flows and its (ex-ante) expected probability of continuation.

P roof. Proposition 2 implies the first assertion as $\beta(l, l)>\beta(l)$ and $\beta(h, l)>\beta(l)$. Next,

$$
\left(1-\theta_{1}\right)^{2} \beta(h, h)+2 \theta_{1}\left(1-\theta_{1}\right) \beta(h, l)+\left(\theta_{1}\right)^{2} \beta(l, l)>\left(1-\theta_{1}\right) \beta(l)+\theta_{1} \beta(l),
$$

holds by $\beta(l, l)>\beta(l)$ surely if $\beta(h, l)=1$, while otherwise it holds from $\beta(h, l)>[1+\beta(l)] / 2$, which follows as in this case $\beta(h, l)=\pi_{h} /\left(2 \bar{\pi}_{2}\right)+\beta(l, l)$. Q.E.D.

Cash-flow pooling (partially) insures a division against being cut off from refinancing following low own cash flows. A higher ex-ante probability of refinancing is also intuitive as the conglomerate uses the 'excess' funds $\pi_{h}-R(h)$ in a more efficient way, i.e., to secure refinancing of the other division. (A $\mathrm{s}$ is easily checked, $R(h, l)$ exceeds the respective payouts of two focused firms.) Finally, the following results follow immediately from Proposition 2.

Corollary 4. If (1) holds, joint incorporation in a homogeneous conglomerate strictly decreases the sensitivity to own cash flows.

Overall, Corollaries 3 and 4 confirm the view that conglomerates are more 'entrenched' as their divisions are more shielded from a shortfall in own cash flows and as the conglomerate as a whole enjoys better access to external finance. However, Corollaries 3 and 4 only hold strictly if $\alpha$ is not too small. Otherwise, i.e., if firms are not financially constrained, there is no difference 
in the investment (and exit or continuation) behavior of focused firms and conglomerates. ${ }^{16}$ Finally, it is noteworthy that the differences made by joint incorporation are entirely due to the independence of first-period cash flows of $A$ and $B$ and not due to the larger size of the conglomerate. To see this, suppose that $A$ and $B$ had perfectly correlated cash flows in $t=1$. By $q(h, l)=0$, none of the effects that derive from cash-flow pooling can be at work and joint incorporation has, consequently, no effects: $\beta(h, h)=\beta(h)$ and $\beta(l, l)=\beta(l)$.

\section{Heterogeneous Divisions}

We now extend the analysis by considering conglomerates with heterogeneous divisions. In particular, to introduce a second key aspect of the internal capital market operated in a conglomerate we specify that one division, say $B$, offers more profitable reinvestment opportunities: $\bar{\pi}_{2}^{B}>\bar{\pi}_{2}^{A}$ as $\theta_{2}^{A}>\theta_{2}^{B}$. Consequently, the firm optimally uses refinancing first for division $B$, i.e., for each cash-flow state $s$ we have that $\beta^{A}(s)>0$ holds only if $\beta^{B}(s)=1$. J oint incorporation thus shields again division $B$ from a shortfall in own cash flows. Intuitively, this has a more pronounced effect on $B$ the more likely it is that $A$ produces high cash flows.

Proposition 3. Suppose B is financially constrained (condition (1)) and has more profitable reinvestment opportunities. Under joint incorporation, B has (i) a higher continuation probability after low own cash flows, (ii) a higher (ex-ante) expected continuation probability, and (iii) a lower sensitivity to own cash flows. The differences to the focused firm decrease with $\theta_{1}^{A}$.

Proof. See A ppendix.

For division $A$ with less profitable reinvestment opportunities, 'winner-picking' in the conglomerate may reverse the findings of Corollaries 3 and 4, making $A$ 'weaker' than if it was operated separately. In what follows, we identify conditions for when this holds. We assume

$$
\pi_{h} \geq \bar{\pi}_{2}^{B}+\bar{\pi}_{2}^{A}
$$

A ssumption (10) ensures that both divisions are refinanced for $(h, l) .{ }^{17}$

\footnotetext{
${ }^{16}$ T hat diversification is more likely to create value if capital markets are less well developed has been found in Comment and J arrell (1995). We are not aware of studies relating differences in exit and investment behavior between conglomerates and focused firms to measures of investor protection or capital market development.

${ }^{17}$ If (10) does not hold, the analysis becomes very tedious and we fail to obtain clear-cut results. The appendix contains a discussion of all cases, showing also that $\beta^{i}(h, l)=1$ always holds if $\alpha$ is not too low or $F$ not too
} 
Proposition 4. Suppose $B$ is financially constrained when operated separately. Then there exists $0<\bar{\theta}<1$ such that under joint incorporation, $A$ has for $\theta_{1}^{B}<\bar{\theta}$ (i) a higher continuation probability after low own cash flows, (ii) a higher (ex-ante) expected continuation probability, and (iii) a lower sensitivity to own cash flows. For $\theta_{1}^{B}>\bar{\theta}$, all three results are reversed.

Proof. See A ppendix.

Proposition 4 questions the general validity of the notion that a conglomerate's divisions are (financially) stronger than respective focused firms. The internal capital market operated in a conglomerate works to the disadvantage of divisions with less profitable reinvestment opportunities. ${ }^{18}$ The dependence of the results in Proposition 4 on $\theta_{1}^{B}$ is again intuitive. For instance, if $B$ always generates high cash flows in $t=1, A^{\prime}$ s funds are never used to repay investors so as to ensure continuation of $B$. Moreover, in case $B$ is not financially constrained, it is also intuitive that $A$ 's refinancing probability would not suffer from joint incorporation.

\section{Discussion: An A nalysis of Predation}

We next apply our results to study the incentives for predation. We proceed in two steps. First, we analyze whether joint incorporation protects a business from predation. Second, we analyze whether a conglomerate's division is more likely to engage itself in predatory activities.

Threat of P redation. We follow once again BS and assume that another firm, $C$, can prey on $A^{\prime}$ 's business in $t=1$. Predation comes at costs $e>0$ to $C$ and increases $A^{\prime}$ 's probability of having low first-period cash flows to $\hat{\theta}_{1}^{A}>\theta_{1}^{A}$. $C^{\prime} s$ profits in $t=2$ increase by $\Delta>0$ if $A$ exits. Suppose first that $A$ is separately incorporated. At the beginning of $t=1, C$ decides whether to prey and $A$ raises fresh funds. ${ }^{19} C^{\prime}$ s incentives to prey depend on $A$ 's cash-flow sensitivity. Given the contracts from Proposition $1, A$ is only secure from predation if

$$
\left(\hat{\theta}_{1}^{A}-\theta_{1}^{A}\right)\left[\beta^{A}(h)-\beta^{A}(l)\right] \Delta \leq e .
$$

high- irrespective of whether (10) is satisfied.

${ }^{18}$ It is again straightforward to show that the firms' joint profits are strictly higher under joint incorporation. B efore raising finance, the owners of $A$ and $B$ would thus optimally merge (and co-manage) their businesses. (See, however, the discussion of strategic effects in Section 5.)

${ }^{19} \mathrm{~A}$ s noted in BS, this is akin to first letting $A$ raise its finances, while contracts can not be credibly revealed to $C$ and, thereby, are of no use as a strategic instrument. As in BS, predation is also non-observable. 
This holds always if $A$ is not financially constrained, while for $\beta^{A}(l)<1$ the 'costs-to-benefits' ratio of predation for $C, e / \Delta$, must not be too low. Likewise, in a conglomerate $A$ is only secure from predation if its expected own cash-flow sensitivity is again not too high:

$$
\left(\hat{\theta}_{1}^{A}-\theta_{1}^{A}\right)\left[\theta_{1}^{B}\left[\beta^{A}(h, l)-\beta^{A}(l, l)\right]+\left(1-\theta_{1}^{B}\right)\left[\beta^{A}(h, h)-\beta^{A}(h, l)\right]\right] \Delta \leq e .
$$

Comparing (11) with (12), we can use our previous analysis of cash-flow sensitivities (Corollary 4 and Propositions 3-4) to obtain the following results. ${ }^{20}$

Proposition 5. Joint incorporation always better protects $A$ from predation if $A$ has better reinvestment opportunities than $B$, i.e., in this case (11) implies (12), but not vice versa. The same holds if $A$ and $B$ are homogeneous. In contrast, if $B$ has better reinvestment opportunities and is financially constrained, there exists $0<\bar{\theta}<1$ such that this holds only for $\theta_{1}^{B}<\bar{\theta}$, while for $\theta_{1}^{B}>\bar{\theta}$ the result is reversed, i.e., (12) implies (11), but not vice versa.

If forming a conglomerate puts $A$ at a competitive disadvantage by making predation more likely, this raises the question why $A$ is not operated separately in the first place. One benefit of joint incorporation is that the other division, $B$, is more protected against predation. In addition, we know that joint incorporation creates strictly positive efficiency gains, which may already more than outweigh any strategic disadvantage in business $A .^{21}$

Incentives for Predation. Suppose now that $A$ can prey on its competitors, in which case $A$ has lower cash flows in $t=1$ but higher cash flows in $t=2$. Precisely, predation leads to an increase from $\theta_{1}^{A}$ to $\hat{\theta}_{1}^{A}$ and to a decrease from $\theta_{2}^{A}$ to $\hat{\theta}_{2}^{A}$. Denote $\hat{\pi}_{t}^{A}:=(1-\hat{\theta}) \pi_{h}$ such that $\hat{\pi}_{1}^{A}<\bar{\pi}_{1}^{A}$ and $\hat{\pi}_{2}^{A}>\bar{\pi}_{2}^{A}$. As in Proposition 5 , we are again interested in when our original

\footnotetext{
${ }^{20}$ For brevity's sake, Proposition 5 stops short of a full equilibrium analysis. If no predation is expected, the values $\beta^{i}(s)$ are clearly optimal such that no-predation is indeed an equilibrium if (11) and (12) hold, respectively. Likewise, no-predation is only an equilibrium if these conditions hold. For a range of parameters, however, multiple equilibria exist. To see this for the focused firm, suppose predation is expected and the resulting financial contract gives rise to values $\hat{\beta}^{A}(s)$, where $\hat{\theta}_{1}^{A}>\theta_{1}^{A}$ implies $\hat{\beta}^{A}(l)<\beta^{A}(l)$ from (4). Predation then pays for $C$ if $\left(\hat{\theta}_{1}^{A}-\theta_{1}^{A}\right)\left[\hat{\beta}^{A}(h)-\hat{\beta}^{A}(l)\right] \Delta \geq e$, implying together with (11) that both predation and no-predation are equilibria for intermediate values of $e / \Delta$. Hence, while predation can only be successful if $A$ is financially constrained, the expectation of predatory activity can itself make financial constraints more severe.

${ }^{21}$ By our previous analysis, these efficiency gains come in two types. First, as found in Section 3 cash-flow pooling allows a more efficient use of 'excess funds' in the state $(h, l)$. Second, 'winner-picking' allows to redirect limited refinancing to the more profitable division, $B$.
} 
contracts support a no-predation equilibrium. But this time it is $A$ that must be prevented from preying. In case of separate incorporation, we find that this is only the case if

$$
\frac{\hat{\theta}_{1}^{A}-\theta_{1}^{A}}{\theta_{2}^{A}-\hat{\theta}_{2}^{A}} \geq \hat{\theta}_{1}^{A} \beta^{A}(l)+\left(1-\hat{\theta}_{1}^{A}\right) \beta^{A}(h) .
$$

(This is formally derived in the proof of Proposition 6.) That is, A's expected refinancing probability after predation, $\hat{\theta}_{1}^{A} \beta^{A}(l)+\left(1-\hat{\theta}_{1}^{A}\right) \beta^{A}(h)$, must be sufficiently low. This is intuitive as $A$ can only benefit from predation if it is refinanced. If $A$ is not financially constrained, as $\beta^{A}(l)=\beta^{A}(h)=1$, condition (13) becomes $\hat{\theta}_{1}^{A}-\theta_{1}^{A} \geq \theta_{2}^{A}-\hat{\theta}_{2}^{A}$, which transforms to $\bar{\pi}_{1}^{A}+\bar{\pi}_{2}^{A} \leq \hat{\pi}_{1}^{A}+\hat{\pi}_{2}^{A}$. That is, a firm that is not financially constrained chooses not not prey only if predation is a negative (or zero) NPV investment. ${ }^{22}$ Consider next a conglomerate where $A$ has more profitable refinancing opportunities, both with and without predation $\left(\hat{\theta}_{2}^{A}<\theta_{2}^{A}<\theta_{2}^{B}\right)$. Given the respective contracts, the conglomerate prefers not to prey only if ${ }^{23}$

$$
\frac{\hat{\theta}_{1}^{A}-\theta_{1}^{A}}{\theta_{2}^{A}-\hat{\theta}_{2}^{A}} \geq \hat{\theta}_{1}^{A}\left[\theta_{1}^{B} \beta^{A}(l, l)+\left(1-\theta_{1}^{B}\right) \beta^{A}(h, l)\right]+(1
$$


Proposition 6. The incentives to prey are always higher for the conglomerate if $A$ has better reinvestment opportunities than B, i.e., (14) implies (13), but not vice versa. The same holds if $A$ and $B$ are homogeneous. If $B$ has better reinvestment opportunities and is financially constrained, we have that: (i) If also $\theta_{2}^{B}-\hat{\theta}_{2}^{A}$ is sufficiently low (in particular, this holds always if $\theta_{2}^{B}<\hat{\theta}_{2}^{A}$ ), there exists $0<\bar{\theta}<1$ such that for $\theta_{1}^{B}>\bar{\theta}$ the result is reversed and a conglomerate has less incentives to prey, while (ii) for high values of $\theta_{2}^{B}-\hat{\theta}_{2}^{A}$ this is not the case $(\bar{\theta}=1)$.

P roof. See A ppendix.

We finally comment on the role of the difference $\theta_{2}^{B}-\hat{\theta}_{2}^{A}$ for the case where refinancing of $A$ is less profitable than refinancing of $B$ without predation but more profitable after predation: $\hat{\theta}_{2}^{A}<\theta_{2}^{B}<\theta_{2}^{A}$. Here, if the firm deviates and preys, this changes the ordering of reinvestment priorities. (The new refinancing probabilities, which we denote by $\hat{\beta}^{i}(s)$, are then simply given by $\hat{\beta}^{A}(s)=\beta^{B}(s)$ and $\hat{\beta}^{B}(s)=\beta^{A}(s)$, reflecting the reversal in preferences.) While we know from our previous results that this leads to a strictly higher probability of refinancing $A$ than in the focused case, there is an important countervailing effect at work. W hile for the focused firm predation increases the total expected payoff from refinancing by $\hat{\pi}_{2}^{A}-\bar{\pi}_{2}^{A}$, the net increase in the conglomerate is smaller: $\hat{\pi}_{2}^{A}-\bar{\pi}_{2}^{B}$. This in turn works in a direction opposite to the increase in $A^{\prime}$ 's refinancing probability, making predation less profitable for the conglomerate.

\section{A ppendix}

Omitted steps for Proposition 2. Denote the constraint that $s$ does not mimic $\widehat{s}$ by

$$
C_{s}^{\widehat{s}}: R(s)-\alpha r(s) \leq 2[\beta(s)-\beta(\widehat{s})](1-\alpha) \bar{\pi}_{2} .
$$

It is straightforward that we can focus on the 'downwards' incentive compatibility constraints. (Formally, after solving the 'relaxed' program it is easily checked that the 'upwards' constraints are satisfied.) As in the proof of Proposition 1, for (1) the lender can only break even if $\beta(l, l)<1$, while we have $R(h, h)<2 \pi_{h}$ and $\beta(h, h)=1$. By the same argument, $\beta(h, l)<1$ can only be optimal if $R(h, l)=\pi_{h}$. We argue next that $C_{h, h}^{l, l}, C_{h, l}^{l, l}$, and $C_{h, h}^{h, l}$ all bind at an optimum. If $C_{h, h}^{h, l}$ does not bind, $C_{h, l}^{l, l}$ implies that also $C_{h, h}^{l, l}$ does not bind, which together with $R(h)<2 \pi_{h}$ allows to marginally adjust $d R(h)>0$, while choosing $d \beta(l, l)>0$ to keep (5) binding and $d R(h, l) \leq 0$ to still satisfy $C_{h, l}^{l, l}$. As is easily seen, this raises the firm's expected payoff by $\left(\theta_{1}\right)^{2} d \beta(l, l) 2\left(\bar{\pi}_{2}-F\right)$. Next, we argue again to a contradiction and assume that 
$C_{h, l}^{l, l}$ does not bind, which by the binding $C_{h, h}^{h, l}$ implies that also $C_{h, h}^{l, l}$ is slack. We marginally adjust $d \beta(h, l)<0$ and choose $d R(h, h)=-d \beta(h, l) 2(1-\alpha) \bar{\pi}_{2}$ to keep $C_{h, h}^{h, l}$ binding. If we set $\left(\theta_{1}\right)^{2} d \beta(l, l)=-2 \theta_{1}\left(1-\theta_{1}\right) d \beta(h, l)$, the firm would be indifferent while the investor's break-even constraint is relaxed by $-d \beta(h, l)(1-\alpha) \bar{\pi}_{2}$, allowing to adjust the contract to increase efficiency. Finally, if $C_{h, l}^{l, l}$ and $C_{h, h}^{h, l}$ bind, also $C_{h, h}^{l, l}$ must bind. Q.E.D.

Derivations for the case with heterogeneous divisions. We maximize $\sum_{s \in S} q(s)[r(s)-$ $\left.R(s)+\sum_{i \in I} \beta^{i}(s)(1-\alpha) \bar{\pi}_{2}^{i}\right]$ subject to the investor's break-even constraint

$$
\sum_{s \in S} q(s)\left[R(s)+\sum_{i \in I} \beta^{i}(s)\left(\alpha \bar{\pi}_{2}^{i}-F\right)\right] \geq 2 F,
$$

the cash-flow constraints $r(s) \geq R(s)$ for $s \in S$, and the set of incentive compatibility constraints. We can rely on the same reasoning as for Proposition 2 to show that: (i) $\beta^{A}(h, h)=\beta^{B}(h, h)=1$ and $r(h, h) \geq R(h, h)$ never binds; (ii) we can restrict consideration to the incentive constraints

$$
R(s)-\alpha r(s) \leq \sum_{i \in I}\left[\beta^{i}(s)-\beta^{i}(l, l)\right](1-\alpha) \bar{\pi}_{2}^{i} \text { for } s \in\{(h, h),(h, l)\},
$$

which are binding at an optimum; and (iii) if $r(h, l) \geq R(h, l)$ does not bind we have $\beta^{i}(h, l)=1$ for $i=A, B$. By an analogous reasoning and using $\bar{\pi}_{2}^{B}>\bar{\pi}_{2}^{A}$, we have that (iv) $\beta^{B}(h, l)=1$ and $\beta^{A}(h, l)>0$. As in Proposition 2, $r(h, l) \geq R(h, l)$ does not bind in Case 1, where $\beta^{A}(h, l)=1$, while it binds in Case 2, where $\beta^{A}(h, l)<1$. Using (17), Case 1 applies if

$$
\pi_{h} \geq \bar{\pi}_{2}^{B}\left[1-\beta^{B}(l, l)\right]+\bar{\pi}_{2}^{A}\left[1-\beta^{A}(l, l)\right]
$$

In Case 1 there are two subcases: $\beta^{A}(l, l)>0$ (Case 1a) and $\beta^{A}(l, l)=0$ (Case 1b). In Case 1a, $\beta^{A}(l, l)>0$ implies $\beta^{B}(l, l)=1$. Using the binding constraints (17) and (16), we obtain

$$
\beta^{A}(l, l)=\frac{\left(\bar{\pi}_{2}^{A}-F\right)\left(1-\theta_{1}^{A} \theta_{1}^{B}\right)-\left(2 F-\alpha \bar{\pi}_{1}^{A}-\alpha \bar{\pi}_{1}^{B}\right)-\left(F-\alpha \bar{\pi}_{2}^{B}\right)}{\left(\bar{\pi}_{2}^{A}-F\right)\left[1-\theta_{1}^{A} \theta_{1}^{B}\right]+\left(F-\alpha \bar{\pi}_{2}^{A}\right)} .
$$

In Case $1 b$, we have $\beta^{A}(l, l)=0$ and obtain from (16)

$$
\beta^{B}(l, l)=\frac{\left[\left(\bar{\pi}_{2}^{B}-F\right)+\left(\bar{\pi}_{2}^{A}-F\right)\right]\left(1-\theta_{1}^{A} \theta_{1}^{B}\right)-\left(2 F-\alpha \bar{\pi}_{1}^{A}-\alpha \bar{\pi}_{1}^{B}\right)}{\left(\bar{\pi}_{2}^{B}-F\right)\left[1-\theta_{1}^{A} \theta_{1}^{B}\right]+\left(F-\alpha \bar{\pi}_{2}^{B}\right)} .
$$

U sing that $\beta^{A}(l, l)>0$ if and only if $\beta^{B}(l, l)=1$, we have that Case 1 a applies if

$$
\left(\bar{\pi}_{2}^{A}-F\right)\left[1-\theta_{1}^{A} \theta_{1}^{B}\right] \geq\left(F-\alpha \bar{\pi}_{1}^{A}\right)+\left(F-\alpha \bar{\pi}_{1}^{B}\right)+\left(F-\alpha \bar{\pi}_{2}^{B}\right) .
$$

For Case 2, recall that by optimality $\beta^{A}(l, l)>0$ only holds if $\beta^{B}(l, l)=1$. But then we can conclude from (18) that in Case 2 it must hold that $\beta^{A}(l, l)=0$. With $R(h, l)=\pi_{h}$, 
constraint (17) for $(h, l)$ becomes next $\pi_{h}=\bar{\pi}_{2}^{B}\left[1-\beta^{B}(l, l)\right]+\beta^{A}(h, l) \bar{\pi}_{2}^{A}$. Solving for $\beta^{A}(h, l)$ and substituting together with (17) for $(h, h)$, we obtain from (16)

$$
\begin{aligned}
& \beta^{B}(l, l)= \frac{\left(\bar{\pi}_{2}^{B}-F\right)\left(1-\theta_{1}^{A} \theta_{1}^{B}\right)+\Pi-\left(2 F-\alpha \bar{\pi}_{1}^{A}-\alpha \bar{\pi}_{1}^{B}\right)}{\left(\bar{\pi}_{2}^{B}-F\right)\left(1-\theta_{1}^{A} \theta_{1}^{B}\right)-\Pi^{\prime}+\left(F-\alpha \bar{\pi}_{2}^{B}\right)}, \text { where } \\
& \Pi \quad: \quad=\left(\bar{\pi}_{2}^{A}-F\right)\left[\left(1-\theta_{1}^{A}\right)\left(1-\theta_{1}^{B}\right)+\left[\theta_{1}^{A}\left(1-\theta_{1}^{B}\right)+\theta_{1}^{B}\left(1-\theta_{1}^{A}\right)\right] \frac{\pi_{h}-\bar{\pi}_{2}^{B}}{\bar{\pi}_{2}^{A}}\right], \\
& \Pi^{\prime} \quad: \quad=\left(\bar{\pi}_{2}^{A}-F\right)\left[\theta_{1}^{A}\left(1-\theta_{1}^{B}\right)+\theta_{1}^{B}\left(1-\theta_{1}^{A}\right)\right] \frac{\bar{\pi}_{2}^{B}}{\bar{\pi}_{2}^{A}} .
\end{aligned}
$$

From (18) we see that Case 2 can not apply if (10) holds, while if (10) does not hold inspection of (22) shows that Case 2 can again be ruled out for high $\alpha$ and low $F$.

Proof of Proposition 3. From the preceding calculations we have for $B$ that $\beta^{B}(h, h)=$ $\beta^{B}(h, l)=1$. In case $\beta^{B}(l, l)=1$ the assertions hold trivially. For $\beta^{B}(l, l)<1$ we consider first Case $1 \mathrm{~b}$, where $d \beta^{B}(l, l) / d \theta_{1}^{A}<0$ holds by (20). To show that $\beta^{B}(l, l)>\beta^{B}(l)$, it is convenient to substitute $\beta^{B}(l, l)=\beta^{B}(l)$ into the investor's payoff for the conglomerate. This yields $\left(1-\theta_{1}^{A} \theta_{1}^{B}\right)\left(\bar{\pi}_{2}^{A}-F\right)-\left(F-\alpha \bar{\pi}_{1}^{A}\right)+\theta_{1}^{B}\left(1-\theta_{1}^{A}\right)\left(\bar{\pi}_{2}^{B}-F\right)\left[1-\beta^{B}(l)\right]>0$. (Recall that $\left(1-\theta_{1}^{A}\right)\left(\bar{\pi}_{2}^{A}-F\right) \geq F-\alpha \bar{\pi}_{1}^{A}$ if $A$ can be financed under separate incorporation.) As the investor's payoff is strictly decreasing in $\beta^{B}(l, l)$, we thus have $\beta^{B}(l, l)>\beta^{B}(l)$. For Case 2 we can proceed analogously to show that $d \beta^{B}(l, l) / d \theta_{1}^{A}<0$ and $\beta^{B}(l, l)>\beta^{B}(l)$. Q.E.D.

Proof of Proposition 4. The difference in probabilities following low own cash flows is

$$
\left(1-\theta_{1}^{B}\right)\left[\beta^{A}(h, l)-\beta^{A}(l)\right]+\theta_{1}^{B}\left[\beta^{A}(l, l)-\beta^{A}(l)\right] .
$$

To see that (23) is strictly decreasing in $\theta_{1}^{B}$, note that $\beta^{A}(l, l)<\beta^{A}(h, l)=1,0<\beta^{A}(l)<1$, and $d \beta^{A}(l, l) / d \theta_{1}^{A} \leq 0$. (For $\beta^{A}(l, l)>0$ this follows from (19).) Moreover, (23) is strictly positive at $\theta_{1}^{B}=0$. At $\theta_{1}^{B}=1$, (23) is strictly negative if and only if $\beta^{A}(l, l)<\beta^{A}(l)$. This holds surely in Case $1 \mathrm{~b}$, where $\beta^{A}(l, l)=0$, while in Case la we can substitute $\theta_{1}^{B}=1$ into (19) and use (4) for $\beta^{A}(l)$ to see that $\beta^{A}(l, l)<\beta^{A}(l)$ holds if and only if $0<\left(F-\alpha \bar{\pi}_{1}^{B}\right)+\left(F-\alpha \bar{\pi}_{2}^{B}\right)$. Next, the difference in ex-ante continuation probabilities is

$$
\begin{aligned}
& \left(1-\theta_{1}^{B}\right)\left[\left(1-\theta_{1}^{A}\right)\left[\beta^{A}(h, h)-\beta^{A}(h)\right]+\theta_{1}^{A}\left[\beta^{A}(h, l)-\beta^{A}(l)\right]\right] \\
& +\theta_{1}^{B}\left[\left(1-\theta_{1}^{A}\right)\left[\beta^{A}(h, l)-\beta^{A}(h)\right]+\theta_{1}^{A}\left[\beta^{A}(l, l)-\beta^{A}(l)\right]\right]
\end{aligned}
$$

while the difference in sensitivities is

$$
\left(1-\theta_{1}^{B}\right)\left[\left(1-\beta^{A}(h, l)\right)-\left(1-\beta^{A}(l)\right)\right]+\theta_{1}^{B}\left[\left(\beta^{A}(h, l)-\beta^{A}(l, l)\right)-\left(1-\beta^{A}(l)\right)\right] .
$$


By $\beta^{A}(l, l)<1$ and $d \beta^{A}(l, l) / d \theta_{1}^{B}<0$, (24) and (25) are strictly increasing in $\theta_{1}^{B}$, while they are again strictly negative at $\theta_{1}^{B}=0$ and strictly positive at $\theta_{1}^{B}=1$ if $\beta^{A}(l, l)<\beta^{A}(l)$. Comparing (23), (24), and (25) shows finally that the thresholds for $\theta_{1}^{B}$ are the same. Q.E.D.

Proof of Proposition 6. Substituting the binding constraint (2), a focused firm's payoff is $(1-\alpha)\left[\bar{\pi}_{1}^{A}+\beta^{A}(l) \bar{\pi}_{2}^{A}\right]$ if (1) holds. If $A$ deviates and preys, its expected payoff is

$$
\hat{\theta}_{1}^{A} \beta^{A}(l)(1-\alpha) \hat{\pi}_{2}^{A}+\left(1-\hat{\theta}_{1}^{A}\right)\left[\pi_{h}-R^{A}(h)+\beta^{A}(h)(1-\alpha) \hat{\pi}_{2}^{A}\right] .
$$

Substituting once more from (2) for $R(h),(26)$ transforms to

$$
(1-\alpha)\left[\hat{\pi}_{1}^{A}+\beta^{A}(l) \bar{\pi}_{2}^{A}+\left[\left(1-\hat{\theta}_{1}^{A}\right) \beta(h)+\hat{\theta}_{1}^{A} \beta(l)\right]\left(\hat{\pi}_{2}^{A}-\bar{\pi}_{2}^{A}\right)\right],
$$

which is not larger than $(1-\alpha)\left[\bar{\pi}_{1}^{A}+\beta^{A}(l) \bar{\pi}_{2}^{A}\right]$ if $\rho:=\left(\hat{\theta}_{1}^{A}-\theta_{1}^{A}\right) /\left(\theta_{2}^{A}-\hat{\theta}_{2}^{A}\right)$ is not smaller than $\rho_{F}:=\hat{\theta}_{1}^{A} \beta^{A}(l)+\left(1-\hat{\theta}_{1}^{A}\right) \beta^{A}(h)$. Take next a conglomerate. Given the contractual choices of $\gamma_{1}(s)$ and $\gamma_{2}(s)$, we denote the firm's optimal refinancing choices by $\beta^{i}(s)$ without predation and by $\hat{\beta}^{i}(s)$ after predation. (Of course, $\hat{\beta}^{A}(s)+\hat{\beta}^{B}(s)=\beta^{A}(s)+\beta^{B}(s)=\gamma_{1}(s)+2 \gamma_{2}(s)$.) Substituting again from (2), the firm's payoff equals

$$
2(1-\alpha)\left[\bar{\pi}_{1}^{A}+\bar{\pi}_{1}^{B}+\beta^{A}(l, l) \bar{\pi}_{2}^{A}+\beta^{B}(l, l) \bar{\pi}_{2}^{B}\right]
$$

without predation and

$$
\begin{aligned}
& \theta_{1}^{B} \hat{\theta}_{1}^{A}(1-\alpha)\left[\hat{\beta}^{A}(l, l) \hat{\pi}_{2}^{A}+\hat{\beta}^{B}(l, l) \bar{\pi}_{2}^{B}\right] \\
& +\left[\hat{\theta}_{1}^{A}\left(1-\theta_{1}^{B}\right)+\theta_{1}^{B}\left(1-\hat{\theta}_{1}^{A}\right)\right]\left[\pi_{h}-R(h, l)+\hat{\beta}^{A}(h, l)(1-\alpha) \hat{\pi}_{2}^{A}+\hat{\beta}^{B}(h, l)(1-\alpha) \bar{\pi}_{2}^{B}\right] \\
& +\left(1-\hat{\theta}_{1}^{A}\right)\left(1-\theta_{1}^{B}\right)\left[2 \pi_{h}-R(h, h)+\hat{\beta}^{A}(h, h)(1-\alpha) \hat{\pi}_{2}^{A}+\hat{\beta}^{B}(h, h)(1-\alpha) \bar{\pi}_{2}^{B}\right]
\end{aligned}
$$

after predation. Suppose now first that $\theta_{2}^{A}<\theta_{2}^{B}$, implying $\beta^{A}(h, l)=\beta^{A}(h, h)=1$ and $\beta^{A}(l, l)>\beta^{A}(l)$ from the proof of Proposition 3. Moreover, we have optimally $\hat{\beta}^{i}(s)=\beta^{i}(s)$. Substituting and comparing (27) with (28), the firm does not deviate and prey if $\rho \geq \rho_{C}:=$ $\theta_{1}^{B} \hat{\theta}_{1}^{A} \beta^{A}(l, l)+\left(1-\theta_{1}^{B} \hat{\theta}_{1}^{A}\right)$, which strictly exceeds $\rho_{F}$. Suppose next that divisions are homogeneous without predation, $\theta_{t}^{i}=\theta_{t}$, implying $\hat{\theta}_{2}^{A}<\theta_{2}^{B}$ such that optimally $\hat{\beta}^{A}(h, h)=$ $\hat{\beta}^{A}(h, l)=1$. Moreover, from Proposition 2 we have $\hat{\beta}^{A}(l, l)=\max \{1,2 \beta(s)\}>\beta^{A}(l)$, where $\beta(s)=\beta^{i}(s)$ is the symmetric refinancing probability without predation. The new threshold $\rho_{C}:=\theta_{1} \hat{\theta}_{1}^{A} \hat{\beta}^{A}(h, l)+\left(1-\theta_{1} \hat{\theta}_{1}^{A}\right)$ satisfies again $\rho_{C}>\rho_{F}$.

Suppose finally that $\theta_{2}^{B}<\theta_{2}^{A}$. If also $\theta_{2}^{B}<\hat{\theta}_{2}^{A}$ holds, predation does not change reinvestment priorities: $\hat{\beta}^{i}(s)=\beta^{i}(s)$. Using $\beta^{i}(h, l)=1$ from (10), we obtain now $\rho_{C}:=\theta_{1}^{B} \hat{\theta}_{1}^{A} \beta^{A}(l, l)+(1-$ 
$\left.\theta_{1}^{B} \hat{\theta}_{1}^{A}\right)$ such that $\rho_{C}>\rho_{F}$ if and only if $1-\beta^{A}(l)>\theta_{1}^{B}\left[1-\beta^{A}(l, l)\right]$. The existence of a threshold $0<\bar{\theta}<1$ such that $\rho_{C}>\rho_{F}$ holds if and only if $\theta_{1}^{B}<\bar{\theta}$ follows then from the proof of Proposition 4. This argument is now easily extended to the case of equality, $\theta_{2}^{B}=\hat{\theta}_{2}^{A}$. If $\theta_{2}^{B}>\hat{\theta}_{2}^{A}$, however, predation changes the reinvestment priorities: $\hat{\beta}^{A}(s)=\beta^{B}(s)$. After substitution to obtain again $\rho_{C}$, we find that $\rho_{C}>\rho_{F}$ holds only if

$$
1-\beta^{A}(l)>\theta_{1}^{B}\left[1-\frac{\left[\beta^{B}(l, l) \hat{\pi}_{2}^{A}+\beta^{A}(l, l) \bar{\pi}_{2}^{B}\right]-\left[\beta^{A}(l, l) \bar{\pi}_{2}^{A}+\beta^{B}(l, l) \bar{\pi}_{2}^{B}\right]}{\hat{\pi}_{2}^{A}-\bar{\pi}_{2}^{A}}\right] .
$$

If $\beta^{A}(l, l)=0$, this transforms to $1-\beta^{A}(l)>\theta_{1}^{B}\left[1-\beta^{B}(l, l)\left(\theta_{2}^{B}-\hat{\theta}_{2}^{A}\right) /\left(\theta_{2}^{A}-\hat{\theta}_{2}^{A}\right)\right]$. This holds surely for $\theta_{1}^{B}=0$, while we know from Proposition 4 that the right-hand side is strictly decreasing in $\theta_{1}^{B}$. It thus remains to show that the inequality holds at $\theta_{1}^{B}=1$ if and only if $\theta_{2}^{B}-\hat{\theta}_{2}^{A}$ is not too small. This finally follows as $\left(\theta_{2}^{B}-\hat{\theta}_{2}^{A}\right) /\left(\theta_{2}^{A}-\hat{\theta}_{2}^{A}\right)$ is strictly decreasing in $\hat{\theta}_{2}^{A}$ (where $\hat{\theta}_{2}^{A}<\theta_{2}^{B}$ ). The case where $\beta^{A}(l, l)>0$ is analogous. Q.E.D.

\section{R eferences}

Benoit, J .-P., Financially Constrained Entry in a Game with Incomplete Information, Rand J ournal of E conomics 15 (1984), 490-499.

Bianco, M. and Nicodano, G., Business Groups and Debt, mimeo, 2002.

Comment, R. and J arrell, G., Corporate Focus and Stock R eturns, J ournal of Financial Economics 37 (1995), 67-87.

B olton, P. and Scharfstein, D.S., A T heory of Predation B ased on A gency Problems in F inancial Contracting, A merican Economic Review 50 (1990), 93-106.

Deily, M., Exit Strategies and Plant-Closing Decisions: The Case of Steel, Rand J ournal of E conomics 22 (1991), 250-263.

Diamond, D., F inancial Intermediation and Delegated M onitoring, R eview of E conomic Studies 51 (1984), 393-414.

Fazzari, S., Hubbard, G., and Petersen, B., Financing Constraints and Corporate Investments, B rookings Papers on E conomic A ctivity 1 (1988), 141-195. 
Fluck, Z. and Lynch, A., Why do Firms Merge and then Divest? A Theory of Financial Synergy, J ournal of Business 72 (1999), 319-346.

Ghemawat, P. and Nalebuff, B., The Devolution of Declining Industries, Quarterly J ournal of Economics 105 (1990), 167-186.

Inderst, R. and M üller, H., Internal versus External Financing: An Optimal Contracting Approach, J ournal of Finance 58 (2003), 1033-1062.

K hanna, N. and Tice, S., The Bright Side of Internal Capital Markets, J ournal of Finance 56 (2001), 1489-1528.

Kovenock, D. and Phillips, G., Capital Structure and Product M arket Behavior: An Examination of Plant Exit and Investment Decisions, Review of Financial Studies 10 (1997), 767-803.

Maksimovic, V., and Phillips, G., Do Conglomerate Firms Allocate Resources Inefficiently A cross Industries?, J ournal of Finance, 75 (2002), 721-767.

Rajan, R., Servaes, H., and Zingales, L., The Cost of Diversity: The Diversification Discount and Inefficient Investment, J ournal of Finance 55 (2000), 35-80.

Scharfstein, D. and Stein, J.C., The Dark Side of Internal Capital Markets: Divisional RentSeeking and Inefficient Investment, J ournal of F inance 55 (2000), 2537-2564.

Stein, J.C., Internal Capital Markets and the Competition for Corporate Resources, J ournal of Finance 52 (1997), 111-133.

Telser, L.G., Cutthroat Competition and the Long Purse, J ournal of Law and Economics 9 (1966), 259-277. 